\title{
Information Model for Estimate of the Geometric Parameters between the Surfaces Trapezoidal Shape in the Measurements of Compressor Blades of GTE
}

\author{
Bolotov M. A., Pechenin V. A., Ruzanov N. V., and Pronichev N. D. \\ Aircraft Engine Faculty, Samara State Aerospace University named after S.P.Korolev (National \\ Research University), 443086, Samara, Russia
}

Keywords: Least square method, compressor blade, assembly.

\begin{abstract}
The paper describes the developed method and the measured information processing model in coordinate measurements of geometric parameters describing the mating surfaces of trapezoidal shape by an example of compressor blades of GTE. The considered pairing includes a blade root and a turbine engine disk slot. Trapezoidal is formed by three planes, including two sides and one lower. The type of mating achieved in the assembly process is considered in the proposed method and the model in the contrast to traditional algorithms and techniques. The results of proposed model testing and the results of the comparison estimates of geometric parameters obtained by using the proposed model and the traditional methods of treatment. We investigate the influence of the errors of form and microroughnesses on accuracy of the estimation of geometrical parameters characterizing the mating surfaces of trapezoidal shape.
\end{abstract}

\section{Introduction}

Measurement and size control are important components of production quality assurance. Measurement results are used to adjust the production equipment and to grade individual parts and assembly units in general. The diversity and complexity of parts and assembly units of engines impose high requirements on flexibility and precision of measuring instruments. In aircraft engine industry the contact and non-contact coordinate measuring machines (CMMs) are often used for the measurement of complex parts and assembly units [1]. Such measuring devices have a high accuracy. However the overall measurement accuracy depends on the data processing algorithms used. In the literature there are many works devoted to the evaluation of the accuracy of geometrical parameters measurements for the individual surfaces such as cylindrical, flat, conical and others [2]. In this paper we consider the accuracy of geometrical parameters characterizing the mating surfaces of trapezoidal shape as an example of compressor blades of GTE.

The mating pair considering in the paper includes root attachment and the slot of a disc of a gas turbine engine. Trapezoidal mating is formed by three planes, including two sides and one lower. In practice of measurements of geometric parameters describing the mating surfaces of trapezoidal shape, there occur cases in which the use of certified measuring tools and algorithms for processing the measured data and measurement techniques do not get accurate results. This is due to the fact that the inherent geometrical parameters calculations methods do not take into account the peculiarities of mating pairs of parts. However, in such measurements aim is to evaluate the accuracy is achieved mates.

\section{Discussed Problems}

The current evaluation of geometrical parameters of mating is carried out in the order. At the first stage coordinate points of surfaces forming the pair are approximated. Geometric parameters characterizing the size and location of surfaces are calculated. In other words this is so-called replacement elements. For example geometric parameters are calculated for the planes, that include the coordinate of point on the plane and the coordinates of the unit director vector. The existing 
approximation algorithms implement evaluation model based on the method of least squares [3] or Chebyshev method or method of described and inscribed elements for cylindrical surfaces.

At the second stage derivatives geometrical parameters characterizing mating are calculated. For example, a trapezoidal blade root mating is characterized by an average right line or a straight plane, which are based on the corresponding right lines or planes. As a result the average line or plane characterizes the mating parts. For compressor blades it results in determining the location of the axis of the blade relative to the axis of the disc.

Determined line or plane sometimes contains significant error in comparison with the actual position reached in the process of pairing [4]. This is due to the necessity of taking into account the mutual influence of the position of the two surfaces when they are paired on a single object, as well as the influence of the form deviation and location deviation of the contact surfaces.

We illustrate the error when the traditional approach is used. During measuring compressor blades root a significant error of the determination of the $\mathrm{Z}$-axis of the blade, which is installed in the compressor disk, is possible (Figure 1).

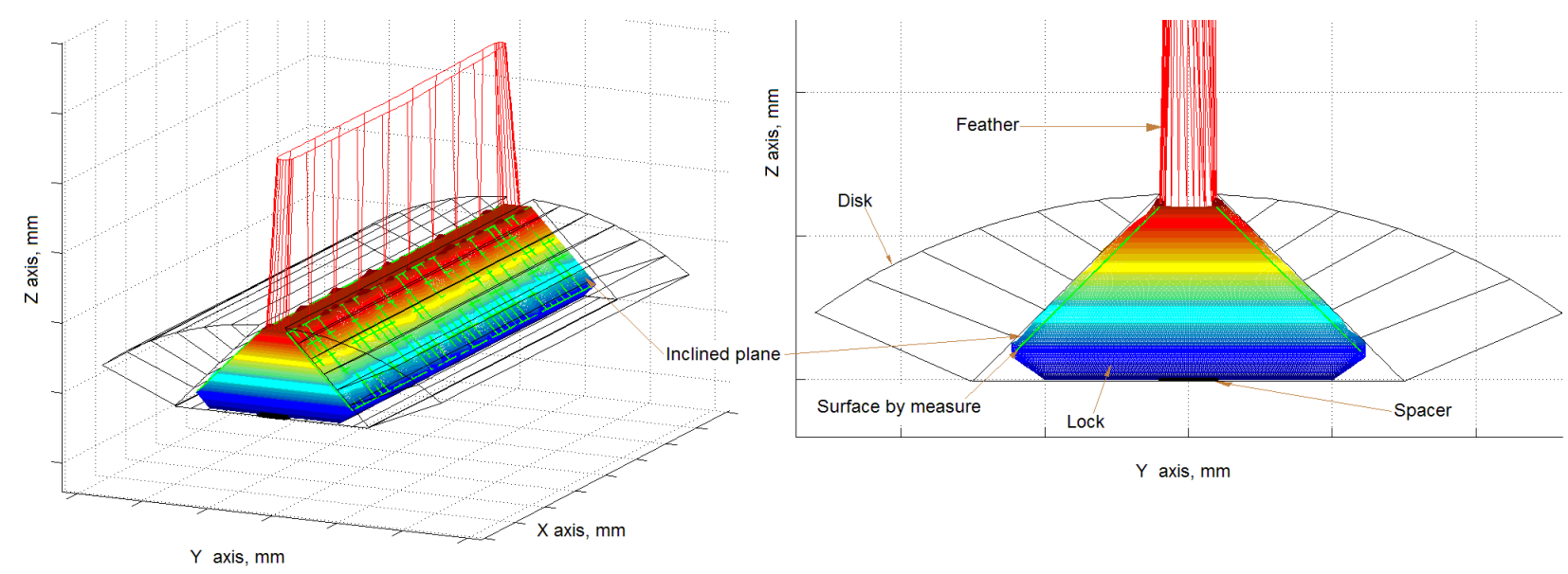

Fig. 1. The shrink fit of the gas turbine engine compressor blade due to the gasket on the lower surface of the root.

The method proposes to count the blade axis taking into account the interactions of contact surfaces of the mating parts and thus it makes the estimate of mating accuracy more sufficient.

\section{Model of Mating Geometrical Parameters Determination}

The Model for Calculating the Geometric Parameters of Mating of Blade Root and Disc Slot GTE. As has been mentioned above we use the model to estimate the geometric parameters of mating covering flat surfaces of the disc slot and covered surfaces, which are represented by compressor blade root. When the side surfaces of the compressor blades trapezoidal root, which have angular error form deviation and microroughness, are contacting with perfectly rigid surface of compressor disc the crumpling of the surface layer of the root side surfaces takes place. During the simulation takes into account the force vector arising from the spacer, which is designed to provide necessary tightness in conjunction after the installation of the blade into the slot. The model is developed in a plane arrangement for cross section of the joint.

The basic assumption of this model is that the groove of the compressor drive (covering part) is absolutely rigid and smooth. In the cross-section surfaces of the slot in which we install the root are two segments with a constant angle between them. The model under consideration is shown in Fig. 2. 


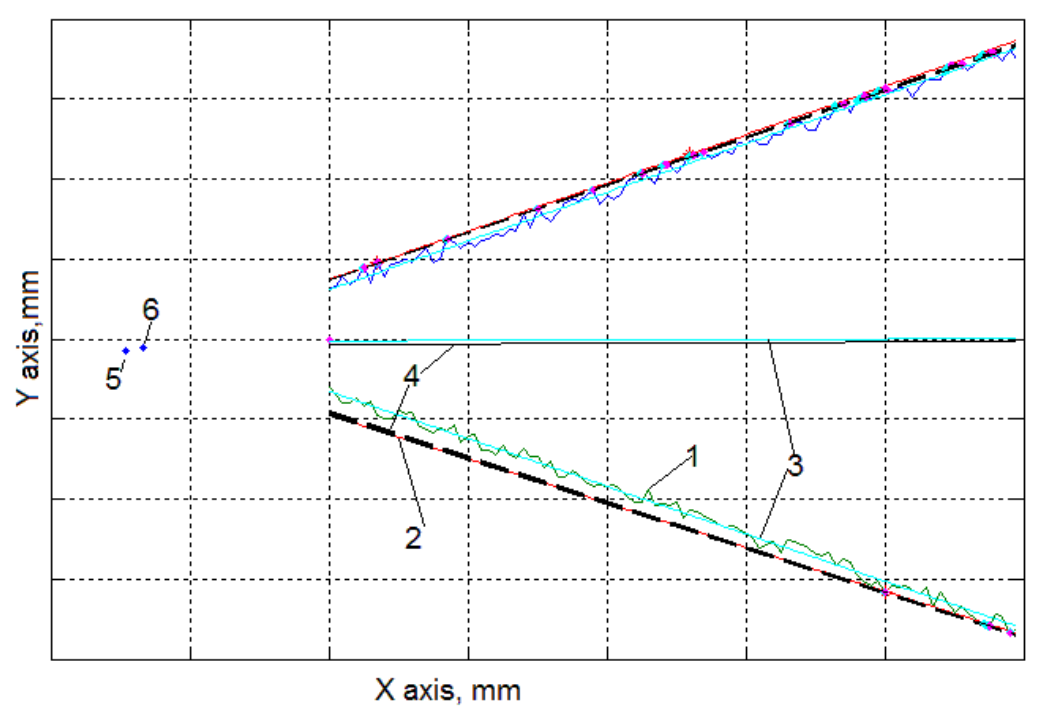

Fig. 2. The cross-sectional approximation model based on the shrink fit of the root in the slot of the disk 1 - the root surface; 2 - position of the slot surface in the moment of contact; 3 - approximation of the surface by OLS, 4 - the final position of the disc slot surfaces with the collapse of root irregularities; 5.6 - initial and final position of the point of intersection of lines 2 and 4.

To set the initial conditions for the optimization algorithm we search for the position of mating parts in model when it is in contact without loading and crush of surfaces. The initial position of the side surfaces of the root is determined by the contact surfaces of the slot at the three points (Fig. 2 circle 3 is initial position of the contacting surfaces of the male and female surfaces of pairs of surfaces).

After the simulation of collapse of root sites irregularities is performed and the mutual arrangement of the male and female surfaces is determined till achievement of tightness, which is determined by the thickness of the spacer (Fig. 2 surface 4).

During assembly the root moves relative to the disk slot. For convenience of calculations in this model it is assumed that the root is fixed and the slot rotates. At the initial moment of contact the coordinates of point 5 (Figure 2)are determined. It is located at the intersection of the lines, which lie on the surface of the slot.

Approximation model is implemented as an iterative algorithm, wherein in each iteration point 5 is shifted along the $\mathrm{x}$-axis at a sufficiently small value. The algorithm stops when the magnitude of the displacement along the $\mathrm{x}$ axis will be equal to the thickness of the spacer.

At each iteration other parameters characterizing the position of the groove surface at each step of the algorithm are calculated such as the coordinate of point 5 along the axis $\mathrm{Y}\left(O_{y}\right)$ and the direction number $k_{1}$ of the upper line of slot surface of the groove. determination of the direction number of bottom line of slot surface is based on value of the number $k_{1}$ and constant angle between the groove $\alpha$ by the fomula:

$k_{2}=\operatorname{tg}\left(\operatorname{arctg}\left(k_{1}\right)-\alpha\right)$.

Absolute term of an equation of lines on which lateral plane of the slot lie are determined using slope coefficient lines and the coordinates of the point $\mathrm{O}$ where these lines intersect.

The determination of values of the required joint parameters is based on the implementation of the objective function:

$$
f\left(O_{y}, k_{1}\right)=\sum_{1}^{n-1}\left|\Delta L_{\text {patch }}^{i+1}-\Delta L_{\text {patch }}^{i}\right| \rightarrow \min ,
$$

where $\Delta L_{\text {patch }}^{i}$ is the increment of the i-th segment length of contact with crumpled.

The objective function describes the condition of equality of increments length of crushing sections in each iteration of the algorithm: 


$$
\Delta L_{\text {patch }}^{1}=\Delta L_{\text {patch }}^{2}=\ldots=\Delta L_{\text {patch }}^{n}
$$

Search for position is conditional nonlinear optimization problem consisting of a nonlinear objective function and linear constraints of its parameters. Optimization problems is solved by the method of sequential quadratic programming (SQP). The method can simulate exactly the Newton method for optimization with constraints [5].

The axis of the blade is defined as a straight line passing through the final position of the point $\mathrm{O}$ and the slope lying on bisector of an angle between the straight sides of the slot.

As mentioned earlier the traditional approach to describing of the problem involves calculating the position of lines that characterize the lateral surface of the blade root by the method of least squares of the measured shaft surface points (Fig. 2, line 3). In the proposed model the blade axis is defined similarly as for the calculation in contact interaction in this way. The coordinates of the point of intersection of the root sides are determined and guiding factor of the axis, which passes through this point, is bisector of lateral angle between the sides.

Estimate of the Error of Geometrical Parameters with the Use of Approximation by OLS. Deviation blade geometry is in relation to the deviation. It is advisable to calculate the deviation of the blade axis points designed at a distance from the blade sole $\left(L_{\mathrm{b}}\right)$, and on surface of its airfoil (Figure 1). As this distance the height of the blade is accepted. Thus the coordinates of a point on the edge of the blade are defined by the equation

$$
M_{2}(x, y)=\left[\begin{array}{ll}
M_{1 x} & M_{1 y}
\end{array}\right]+L_{b} \cdot\left[\begin{array}{ll}
n_{x} & n_{y}
\end{array}\right],
$$

where $M_{1}, M_{2}$ are the start and end point of the height of the blade;

$\left[\begin{array}{ll}n_{x} & n_{y}\end{array}\right]$ are calculated coordinates of the direction vector of the axis of the blade.

The coordinate of point on the axis of the blade obtained by LSO is calculated similarly ( $M_{\mathrm{LSQ}}$.). We define the distance between the points $M_{2}$ and $M_{\mathrm{LSQ}}$. The absolute value of the difference between the geometry by LSO and the proposed method is defined by:

$$
\delta_{a b s}=\left\|M_{2}-M_{\mathrm{LSQ}}\right\| \text {. }
$$

For description of the abnormalities occurring on the set of blades the reduced deviation must be used. The relative deviation can be calculated by the next formula:

$$
\delta_{a b s}=\left\|M_{2}-M_{\mathrm{LSQ}}\right\| / L_{b} \text {. }
$$

\section{Computational examples and Analysis}

The developed model was tested on a set of root profiles having an error of inclined plane and error of side surfaces and form deviation in the range of $0.022 \mathrm{~mm}$. Using the expression (5) the deviation

\begin{tabular}{|c|c|c|c|c|}
\hline & & \multicolumn{3}{|c|}{ Inclined plane } \\
\hline & & $60^{\circ}$ & $59^{0} 56^{\prime}$ & $59^{0} 52^{\prime}$ \\
\hline \multirow{6}{*}{ 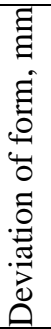 } & 0.0020 & 0.0027 & 0.0409 & 0.0828 \\
\hline & 0.0060 & 0.0077 & 0.0499 & 0.0892 \\
\hline & 0.0100 & 0.0106 & 0.0527 & 0.0894 \\
\hline & 0.0140 & 0.0063 & 0.0361 & 0.0676 \\
\hline & 0.0180 & 0.0227 & 0.0186 & 0.0614 \\
\hline & 0.0220 & 0.0387 & 0.0089 & 0.0571 \\
\hline
\end{tabular}
of coordinates of points on the blade shelves was defined (the distance from the plane of the shank is about 4 heights of the shank ).

Table I: The absolute values of errors in determining the point coordinates on the edge of the blade by LSO, mm 
In order to compare deviations arising on different types of compressor blades we use the expression (6). The relative magnitudes of the calculated errors are shown in Table 2.

Table II: The relative values of errors in determining the coordinates of points on the edge of the blade

\begin{tabular}{|c|c|c|c|c|}
\hline & & \multicolumn{3}{|c|}{ Inclined plane } \\
\hline & & $60^{\circ}$ & $59^{0} 56^{\prime}$ & $59^{0} 52^{\prime}$ \\
\hline \multirow{6}{*}{ 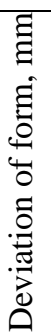 } & 0.0020 & 0.0000 & 0.0005 & 0.0009 \\
\hline & 0.0060 & 0.0001 & 0.0006 & 0.0010 \\
\hline & 0.0100 & 0.0001 & 0.0006 & 0.0010 \\
\hline & 0.0140 & 0.0001 & 0.0004 & 0.0008 \\
\hline & 0.0180 & 0.0003 & 0.0002 & 0.0007 \\
\hline & 0.0220 & 0.0004 & 0.0001 & 0.0006 \\
\hline
\end{tabular}

The magnitude of deviations increases significantly when deviation of root mating angle increases. The influence of form deviation increase on error is less clear and less great. Probably we can conclude that the main role plays not the absolute value but the nature of the irregularities. Position of the axis of the blade calculated by the method of least squares is quite different from when position achieved during the simulation. Especially the difference is large when interference is relatively a small amount.

\section{Conclusions}

The paper proposes a model for evaluation of geometric parameters of trapezoidal surfaces mating which are presented as the sides of the blade root and the disc slot. The model accounts for conjugation between root surfaces and a groove in the disc during its contact. The proposed model and the method has a high efficiency. Using the proposed approach, which accounts for the mating surface and contact interaction, as a base the universal algorithm or series of algorithms can be developed. Algorithms will increase the accuracy of the quality assessment. It is required to test algorithms by physical modeling and experimental studies that reproduce the assembly process.

\section{Acknowledgements}

This work was performed on the equipment of the common use center "CAM-technology".

\section{References}

[1] Savio, E., De Chiffre L., Schmitt R. Metrology of freeform shaped parts. Elseiver. pp.810-835. 2007.

[2] Kamali-Nejad M, Vignat F, Villeneuve F. Simulation of the geometrical defects of manufacturing. International Journal of Advanced Manufacturing Technology. 45(7). pp.631-648.2009.

[3] A. Kawalec, M. Magdziak. Usability assessment of selected methods of optimization for some measurement task in coordinate measurement technique. Measurement. 45. pp.2330-2338. 2012.

[4] Samper, S. Adragna, P.-A., Favreliere, H., Pillet, M. Modeling of 2D and 3D assemblies taking into account for errors of plane surfaces. Journal of Computing and Information Science in Engineering. 9 (4). pp. 041005. 2009.

[5] Powell M.J.D. A fast algorithm for nonlinearly constrained optimization calculations, numerical analysis. ed. G.A. Watson, Lecture Notes in Mathematics, Springer Verlag. 1978. 